\title{
Single-sided interferometric EMIR method for NDE of structures
}

\author{
by $P$. Levesque and D. L. Balageas
}

ONERA, Département DMSE, 29, av. de la Division Leclerc, BP 72, 92300 Châtillon Cedex, France levesque@onera.fr, balageas@onera.fr

\begin{abstract}
Recent work at ONERA demonstrates the interest of using microwave interferometry associated with the EMIR (ElectroMagnetic-InfraRed) method in non destructive evaluation. Until now the method was based on the doubled-sided configuration which is not always applicable in industrial conditions. A single-sided new interferometric method is presented here which avoids this drawback.
\end{abstract}

\section{EMIR (ElectroMagnetic-InfraRed) method theory}

To provide a simple means of measuring an electromagnetic (EM) field, ONERA $[1,2]$ has developed a sensor consisting of a thin film whose electrical (or magnetic) properties are such that it converts a part of the incident field into heat. The film is very thin (some tens of microns), which limits the lateral heat conduction, improving the spatial resolution. Its emissivity is chosen to maximize the infrared energy received by the infrared camera.

The dissipated volume power density is given by the well-known formula:

$$
\left.P_{a h s}=0.5\left[\left(\sigma+\varpi \varepsilon^{\prime \prime}\right) E^{2}+\varpi \mu^{\prime \prime} H^{2}\right]=P_{a b s}=0.5 \mid\left(\sigma_{e q}\right) E^{2}+\varpi \mu^{\prime \prime} H^{2}\right]
$$

in which $\sigma$ is the conductivity of the material, $\varepsilon=\varepsilon^{\prime}-j \varepsilon^{\prime \prime}$ its complex permittivity, and $\mu=\mu^{\prime}-j \mu^{\prime \prime}$ its complex permeability, $\mathrm{E}$ and $\mathrm{H}$ being the amplitudes of the electric and magnetic incident fields.

The resulting temperature rise depends not only on the absorbed energy, but also on the thermal properties of the film and on the heat transfer with the surroundings as well. To decrease the influence of the convection and radiation losses, an amplitude modulation of the EM field associated with a lock-in thermographic detection was proposed $[3,4]$. This technique increases the dynamic range and the spatial resolution when the modulation frequency is increased, but at the same time the signal-to-noise ratio is degraded. A modulation frequency of $1 \mathrm{~Hz}$ is a good compromise.

The technique described above only permits amplitude mapping of the EM field. To measure the phase, ONERA has developed since 1994 a holographic method in which two coherent plane waves are interfering, one of them being used as the reference $[5,6]$. It is a double-sided method, the two needed antennas or the antenna and an EM mirror being respectively on each side of the inspected structure. For NDE, the tested sample is located near of the film. Previous work used both single-sided and double-sided configurations with a unique antenna. These configurations are presented in Fig. 1 and their adequacy for the NDE of various types of EM materials are summarized. They are compared to the new technique here presented.

\section{Principle of the single-sided interferometric EMIR method}

The single-sided interferometric method consists of generating at the photothermal film located close to the front face of the tested structure interference between two coherent plane waves emitted by two antennas located in front of the structure. The resulting field of interference is then visualized by an infrared camera mapping the film temperature distribution. This film temperature increase is proportional to the intensity of the electric field. 
As in the double-sided method, it is possible by phase-stepping operations to map the phase distribution in the film plane as well.

The detection of defects in the sample is done directly by the observation of the modifications of the interfering system in interaction with the sample via the reflected field $E_{r}$.

It is possible to find planes in which the interference field has a constant phase and the intensity is maximum. These planes will be ideal for defect detection, since for a sound tested sample, with low reflection coefficient, the defect presence will creates in the IR image of the film a local contrast in a homogeneous background.

Let us consider the interferometric arrangement using two antennas (see Fig. 2), with no sample under test. Let us assume, for simplification, that the electric fields of the antennas are perpendicular to the direction of propagation and vertically polarized. At a point $M(x, y, z)$ the intensity of the two incident electric fields is, with $\bar{r}=(x, y, z)$ :

$$
I(x, y, z)=E_{1}^{2}(x, y, z)+E_{2}^{2}(x, y, z)+2 E_{1}(x, y, z) E_{2}(x, y, z) \cos (\Delta \vec{k} \vec{r}+\Delta \Phi)
$$

There is a constructive interference when: $\Delta \vec{k} \vec{r}+\Delta \Phi=2 n \pi$

Expression (2) allows the definition of the positions of the planes in which the phase is constant and the total intensity of the field due to the two antennas is maximum:

$$
x=-n \lambda / \sin (\theta)+\Delta \Phi \lambda / 2 n \sin (\theta)+\tan (\theta / 2) z
$$

It is found that resulting isophase planes are inclined by an angle $\theta / 2$ from the $\mathrm{Oz}$ axis. The distance between two successive isophase planes (180 ${ }^{\circ}$ phase lag), following respectively the $\mathrm{Ox}$ and $\mathrm{Oz}$ directions, are given by the expressions:

$$
\begin{gathered}
\left|x_{n}-x_{n+1}\right|=i_{x}=\lambda / \sin \theta \\
\left|z_{n}-z_{n+1}\right|=i_{z}=\lambda /(1-\cos \theta)
\end{gathered}
$$

From isophase plane to isophase plane, the intensity varies. Of course it is preferable to locate the film in a region of maximum intensity. For a microwave frequency of $14 \mathrm{GHz}$ $(\lambda=2,142 \mathrm{~cm})$ and $\theta=30^{\circ}$, we obtain $i_{x}=4,284 \mathrm{~cm}$ and $i_{z}=15,98 \mathrm{~cm}$. Fig. 3 compares the amplitude and phase distribution of the electric field in an isophase plane and in a plane set in any part of the interference field. In this last case fringe distance is $6.4 \mathrm{~cm}$.

The phase images result from the combination of four successive intensity images obtained with each antenna alone, the two antennas working concomitantly with and without a relative phase lag of $\pi / 2$.

\section{Application to non-destructive testing}

Four examples will be given to demonstrate the ability of this method in the detection of defects. An Agema 880 camera associated with a lock-in system is used. The modulation frequency was $1 \mathrm{~Hz}$ and the number of integrated images was 500 . The microwave frequency used was $14 \mathrm{GHz}$. Schematic configuration is shown on Fig. 4. The photothermal film was placed on a isophase plane in which the phase was quasi uniform especially for homogenous radar absorbing materials with which the reflected field is very weak. The tested sample was moved in front of the film and the presence of a defect generated a modification of the interfering fields, in particular in the film plane. This was detected by the infrared camera.

The first example concerns a radar absorbing material LS30 (sample dimensions $\left.300 \times 300 \times 9 \mathrm{~mm}, \varepsilon_{\mathrm{r}}=2.4-\mathrm{j} 15\right)$ containing a defect made of a different absorber, LS20 $\left(\varepsilon_{\mathrm{r}}=1.5-\mathrm{j} 0.69\right)$. The defect is square shaped, $20 \times 20 \mathrm{~mm}$, with a thickness of $3 \mathrm{~mm}$, located at a depth of $3 \mathrm{~mm}$ from the surface. Fig.s 5 and $A$ shows the total field measured in 
presence and absence of the defect. The defect occurrence is visible in both amplitude and phase images, and this is particularly true when the reflected field is weak.

The second example concerns a homogeneous sample (of dimensions $300 \times 300 \times 20 \mathrm{~mm}$ ) of radome material, of permittivity equal to 3.5 and presenting on its back side an overthickness of $10 \mathrm{~mm}$ of dimensions $10 \times 10 \mathrm{~mm}$. Fig. 6 shows the resulting EMIR images with and without defect. We notice the presence of interferences even without overthickness. This is due to the field reflected by the sample itself. The detection of the defect is in this example more complex than in the preceding one as shown on fig. 6 (right), which represents the subtraction of the pictures obtained with and without defect. Nevertheless, we will note that the classic method via reflection could not have helped find this defect in real time.

The nature and the dimension of the defect influence the changes of the interference field especially when the photothermal film is located on an isophase plane corresponding to an extremum of intensity of the electric field. To illustrate this fact, the results obtained for the same radome sample but with a $7 \mathrm{~mm}$-side square metallic insert, located at a depth of 15 $\mathrm{mm}$, are presented in fig. 7. The photothermal film is located either on the minimum of the electric field (see fig. 7 , left) or on a maximum (fig. 7, center). These results demonstrate that, with interferometry, it is possible to adjust the phase lag to enhance the contrast due to the defect.

Fig. 7-right represents the subtraction of the images of the reference sample without defect when the photothermal film is located on the maximum of the electric field. We can notice that the defect and its position appear clearly here.

The last example concerns a weak conductor sample of thickness $15 \mathrm{~mm}$ and dimensions $300 \times 300 \mathrm{~mm}$, made of carbon fibers in a resin matrix, with a volume fiber content of $33 \%$. The sample generates an important reflected field visible on fig. 8 , left. This sample presents on its surface a square-shaped hole of dimensions $5 \times 5 \mathrm{~mm}$ and $5 \mathrm{~mm}$ depth. The presence of the defect can be clearly detected as presented in fig. 8 (center). This singlesided interferometric method, although not well adapted to materials with a strong coefficient of reflection, is interesting in the present case, as shown by the differential image presented in fig. 8 (right). The location of the defect is clearly revealed with a good spatial resolution.

\section{Conclusion}

From these examples, a qualitative demonstration is given of the ability of the singlesided interferometric method to detect, in real time, the presence of defects in various types of materials, such as electromagnetic window materials and radar absorbers. This method is nevertheless especially interesting for materials having a weak coefficient of reflection (more than $20 \mathrm{~dB}$ ) like radar absorbing materials. In that sense, it appears as complementary of the double-sided interferometric technique which is more suitable for dielectric materials (see comparison in fig. 1). The results also confirm the interest of interferometric methods compared to intensity techniques using a unique antenna. For a quantitative diagnostic on the shape and size of the defect and the real sensitivity of the method, it will be necessary to develop theoretical calculations taking into account the reflected fields and to compare them to experimental data. Defects of smaller dimensions (a few millimeters) could have been detected by increasing the microwave frequency up to $35 \mathrm{GHz}$ for example. Among advantages of this method, are its easy applicability to axisymmetrical structures as is obvious by rotation of the tested structure. One of the potential applications of this method is the detection of objects buried in the ground.

\section{REFERENCES}

[1] BALAGEAS (D. L.), LEVESQUE (P.), NACITAS (M.), KRAPEZ (J.-C.), GARDETTE (G.) Photothermal mapping of electromagnetic fields: state-of-the-art, Progress in Natural Sc. (China), Suppl. to vol. 6, 1996, p. 677-684. 
[2] LEVESQUE (P.), DÉOM (A.) and BALAGEAS (D. L.) - NDE of absorbing materials using microwave stimulated infrared thermography, QIRT 92, ed. D. Balageas, G. Busse, G.M. Carlomagno, Editions Europ. Thermique et Industrie, Paris, 1992, p. 302-307.

[3] BALAGEAS (D. L.), LEVESQUE (P.) and DÉOM (A.) - Characterization of electromagnetic fields using lock-in IR thermography, Thermosense XV, SPIE vol. 1933, 1993, p. 274-285.

[4] NACITAS (M.), LEVESQUE (P.), BALAGEAS (D. L.) - Lock-in thermography applied to the characterization of electromagnetic fileds, QIRT 94, ed. D. Balageas, G. Busse, G.M. Carlomagno, Editions Europ. Thermique et Industrie, Paris, 1995, p. 287-292.

[5] BALAGEAS (D. L.), LEVESQUE (P.), NACITAS (M.), KRAPEZ (J.-C.), GARDETTE (G.) Microwaves holography revealed by photothermal films: application to NDE of dielectric and radar absorbing materials, QIRT 96, ed. D. Balageas, G. Busse, G.M. Carlomagno, Edizioni ETS, Pisa (Italy), 1997, p. 73-78.

[6] BALAGEAS (D. L.), LEVESQUE (P.), NACITAS (N.), KRAPEZ (J.-C.), GARDETTE (G.), LEMISTRE (M.) - Microwaves holography revealed by photothermal films and lock-in IR thermography: application to electromagnetic materials NDE, SPIE Proc. 2944, SPIE, 1996, p. 55-66.

[7] BALAGEAS (D.), LEVESQUE (P.) - EMIR: a photothermal tool for electromagnetic phenomena characterization, Rev. Gén. Therm., 37, 8,1998, p. 725-739.

\begin{tabular}{|c|c|c|c|c|}
\hline & \multicolumn{2}{|c|}{ Single-sided method } & \multicolumn{2}{|c|}{ Double-sided method } \\
\hline & Antenna alone & Interferometric & Antenna alone & Interferometric \\
\hline $\begin{array}{l}\text { Schematic } \\
\text { configuration }\end{array}$ & & & 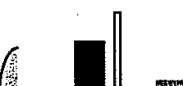 & 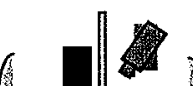 \\
\hline $\begin{array}{l}\text { Radar absorbing } \\
\text { materials }\end{array}$ & + & + & & \\
\hline $\begin{array}{l}\quad \quad \\
\text { Electromagnetic } \\
\text { window } \\
\text { materials }\end{array}$ & + & $\begin{array}{l}1 \\
\ddots\end{array}$ & $\begin{array}{l}1 \\
+\end{array}$ & $\begin{array}{c}1 \\
++\end{array}$ \\
\hline
\end{tabular}

Fig. 1: Various possible configurations for NDE by EMIR. Comparative advantages. 
http://dx.doi.org/10.21611/qirt.1998.004

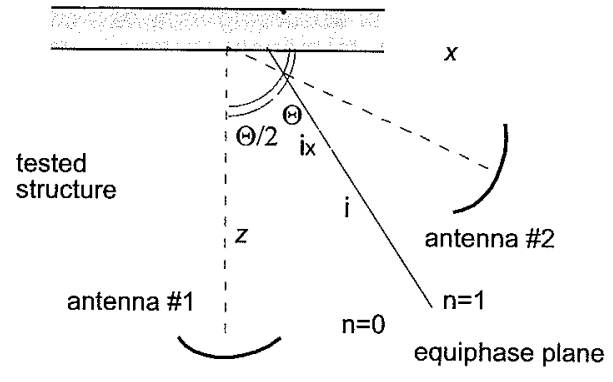

Fig. 2. Principle of the single-sided method. Isophase planes

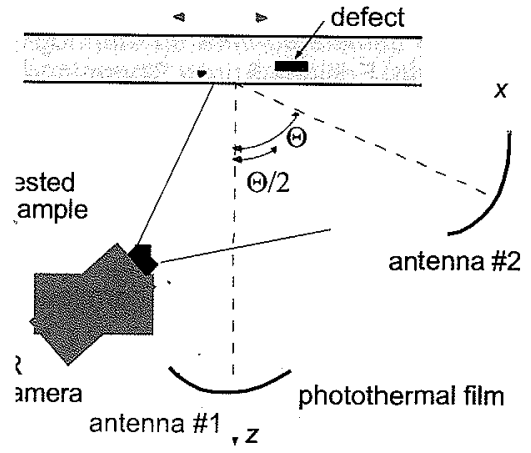

Fig. 4. Schematic NDE configuration for single-sided interferometric EMIR method

amplitude images
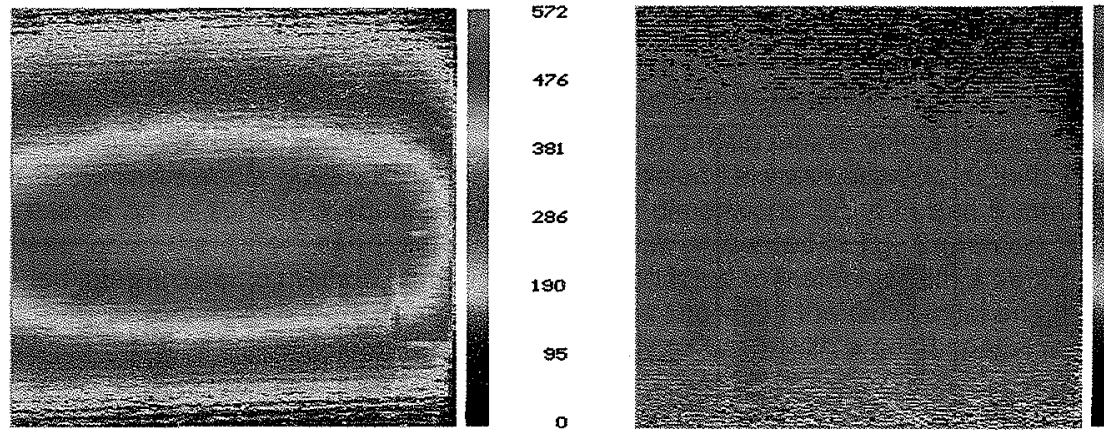

360

300

$2+0$

180

120

60

phase images

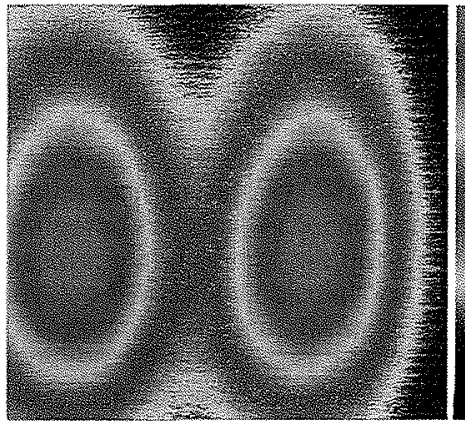

a)

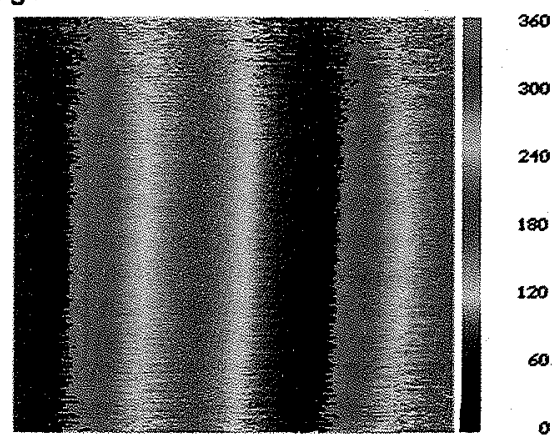

b)

Fig. 3. Amplitude (V/m) and phase $\left({ }^{\circ}\right)$ EMIR images. a). Photothermal film is located in an isophase plane; b) Photothermal film is set in any location. 
http://dx.doi.org/10.21611/qirt.1998.004

amplitude images
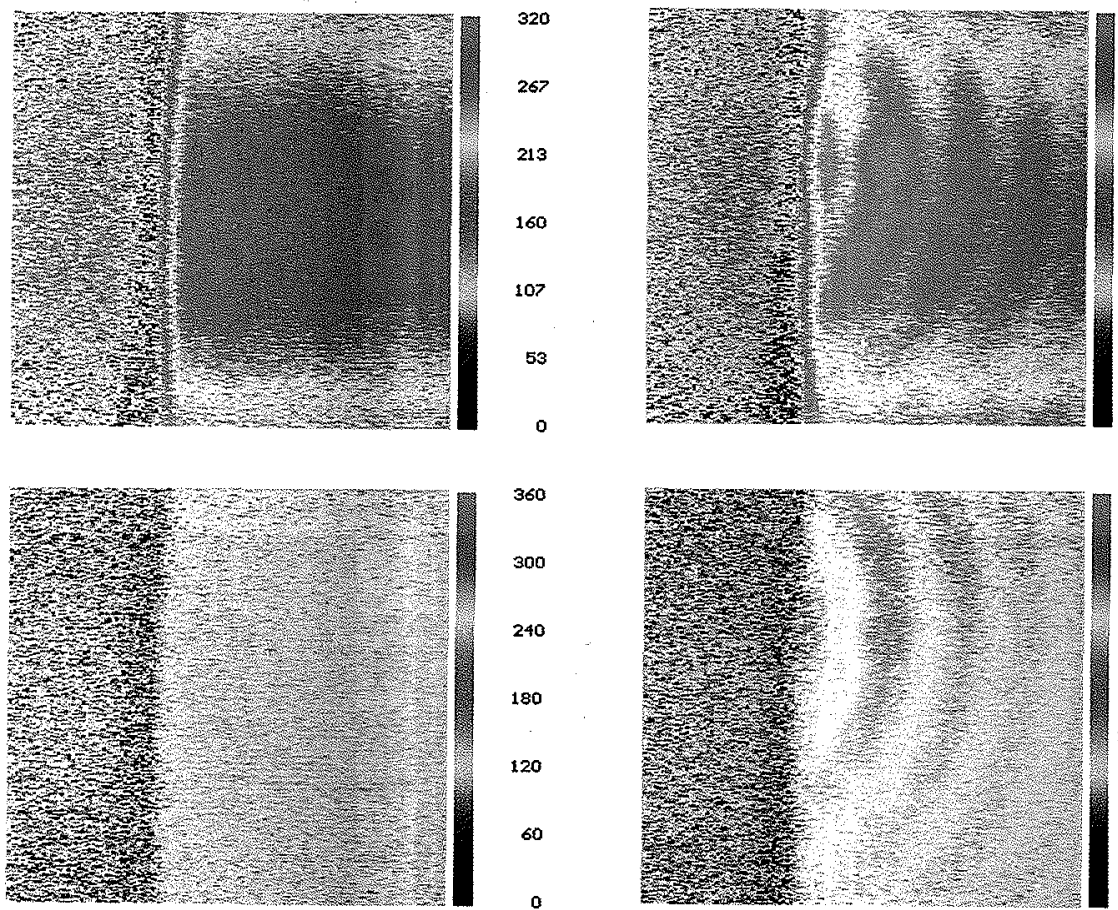

360

sound region

defect region

Fig. 5. Detection of a defect in a radar absorbing sample
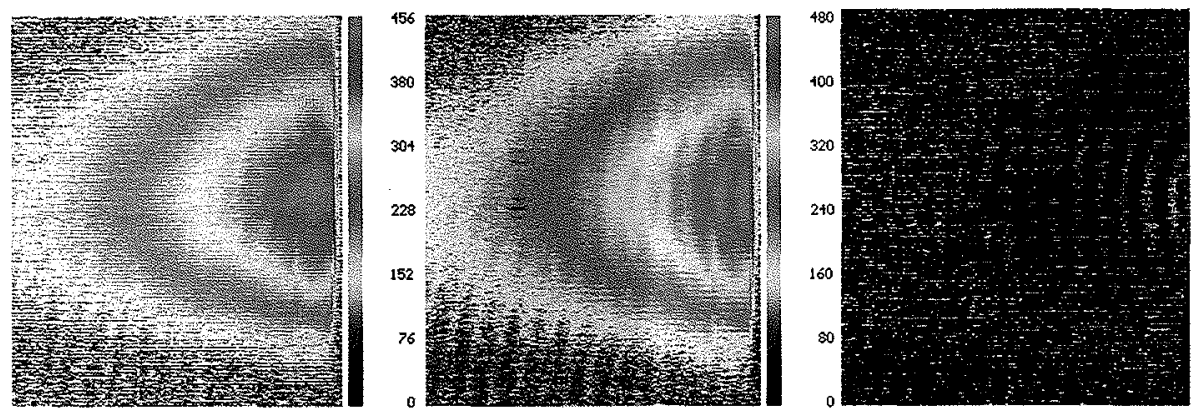

Fig. 6. Radome sample with overthickness. From left to right: E field amplitude in front of regions with and without overthickness and differential image. 
http://dx.doi.org/10.21611/qirt.1998.004
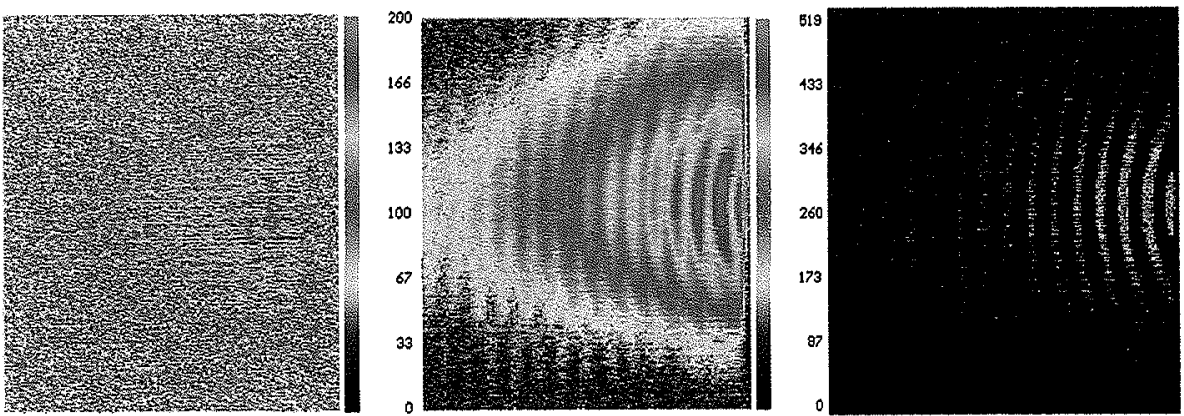

Fig. 7. Radome sample with metallic insert. From left to right: intensity image on a minimum of field, idem on a maximum, and subtraction from the last image of the image of the sample without defect.
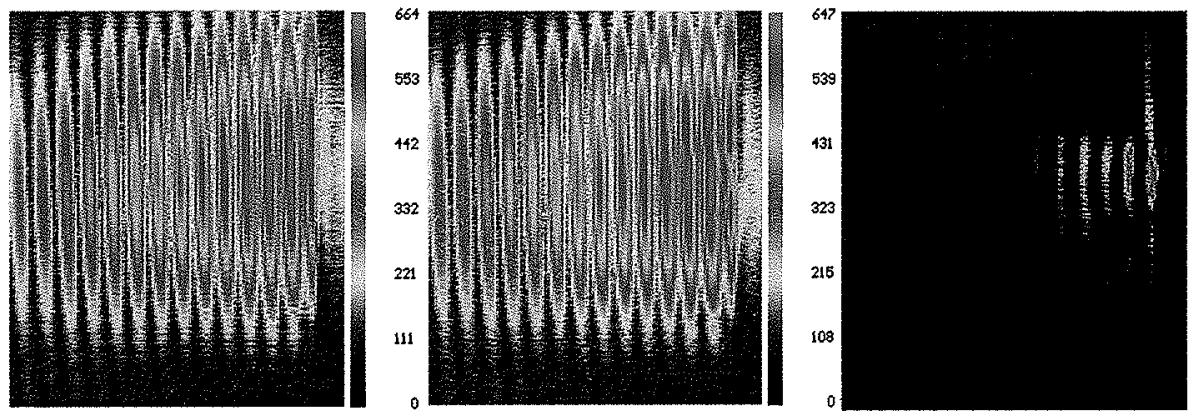

Fig. 8. Composite with carbon fibers. From left to right: sample without and with defect and differential image. 